\title{
Mobisaic: An Information System for a Mobile Wireless Computing Environment
}

\author{
Geoffrey M. Voelker and Brian N. Bershad \\ Department of Computer Science and Engineering \\ University of Washington \\ Seattle, WA 98195
}

\begin{abstract}
Mobisaic is a World Wide Web information system designed to serve users in a mobile wireless computing environment. Mobisaic extends the Web by allowing documents to both refer and react to potentially changing contextual information, such as current location in the wireless network. Mobisaic relies on clientside processing of HTML documents that support two new concepts: Dynamic Uniform Resource Locators (URLs) and Active Documents. A dynamic URL is one whose results depend upon the state of the user's mobile context at the time it is resolved. An active document is one that automatically updates its contents in response to changes in a user's mobile context. This paper describes the design of Mobisaic, the mechanism it uses for representing a user's mobile context, and the extensions made to the syntax and function of Uniform Resource Locators and HyperText Markup Language documents to support mobility.
\end{abstract}

\section{Introduction}

This paper describes Mobisaic, a system that uses the World Wide Web [Berners-Lee et al. 92] to enable information browsing in a mobile computing environment. Information browsing is an ideal mobile application because it allows users to interact with their environment as they work within it, it places minimal requirements on a user-input device, and it cannot be handled with large on-board caching. With mobile information browsing, users can discover who and what is in their immediate surroundings, whether it is colleagues at a business meeting, speakers at a presentation, projects in a lab, or displays in a museum. A mobile information system can also allow users to execute general queries that incorporate information from their environment, such as finding the nearest cafe or the nearest bus stop that will take them to a specific location.

This research was sponsored by a National Science Foundation Presidential Young Investigator Award, together with matching funds from the Xerox Corporation.
Users of the World Wide Web rely on client browsers to access information servers on the Internet. With Web clients, users browse documents written in the HyperText Markup Language (HTML) by traversing hypertext links, called Uniform Resource Locators (URLs), that load documents or invoke programs on the information servers.

Mobisaic extends standard client browsers to take advantage of mobility in two ways. First, Mobisaic allows authors to reference dynamic information, such as a user's location, in hypertext links called dynamic URLs. When the user traverses a dynamic URL, the client resolves any references to dynamic information it may contain and sends the result to the server. Second, Mobisaic supports active documents, documents that present and automatically update information for the user as the information they contain changes or otherwise becomes invalid. The update is done by the client browser, which receives notifications when the dynamic information changes.

Dynamic information in Mobisaic is represented using dynamic environments [Schilit et al. 93B]. Just as standard shells provide environment variables to customize applications started from the shell (e.g., DISPLAY), dynamic environments provide environment variables for customizing mobile applications. For example, a dynamic document might include a reference to the Location dynamic environment variable to customize its contents according to the user's current location.

\section{$1.1 \quad$ Related work}

Researchers at Xerox PARC have broadly introduced the idea of context-aware applications [Schilit et al. 93A]. They initially proposed the notion of dynamic environments as a means of representing and disseminating information from users' mobile contexts throughout the system. Mobisaic is essentially an application of those ideas to WWW browsing. Projects at DEC WRL and MIT have moved the World Wide Web client interface to a mobile device, although they do not appear to incorporate informa- 
tion from the user's mobile computing context into the system.

\subsection{Paper outline}

The rest of this paper is organized as follows. Section 2 gives an overview of the World Wide Web system, and describes the extensions to the system used for incorporating a user's mobile computing context into the WWW. Sections 3 and 4 describe dynamic URLs and active documents. Section 5 discusses how Mobisaic can be useful in the desktop environment as well as the mobile environment. Section 6 describes the implementation of Mobisaic. Section 7 summarizes.

\section{System overview}

This section first provides a high-level overview of the World Wide Web information system, and then describes the extensions used for incorporating a user's mobile computing environment into the system.

\subsection{The World Wide Web}

The three main components of a World Wide Web (WWW) information system are documents, clients, and information servers. The user interacts with the system using a Web client, which lets the user name and load documents from servers for viewing. Web clients typically support a number of different document types, such as ftp, netnews, and Hypertext Markup Language (HTML), and support connections with a variety of information servers, such as ftp daemons, news servers, and Hypertext Transport Protocol (HTTP) daemons. Documents are typically files on a server referenced by Uniform Resource Locators (URLs), and they can contain a variety of information types, including ASCII text formatted according to HTML directives, embedded pictures, and audio and video clips, as well as embedded URLs that are used as hypertext links to other documents. URLs can also name programs on HTTP daemons that, when executed, produce an HTML document as output.

\subsection{Extensions for a mobile WWW}

In its current form, the Web infrastructure cannot easily accommodate mobile clients because information is either statically expressed in HTML documents, or because all non-static information must be explicitly entered through form-based interfaces that run on the client.

In its current form, the Web infrastructure cannot easily accommodate mobile clients because the dynamic information it supports is either returned from the server without incorporating any user context at all, or is incorporated explicitly using forms-based interfaces that require user input on the client. Moreover, there is no support for automatically updating a document when it, or the reason for displaying it, changes.

To better support the use of dynamic information, we have extended the Web infrastructure to include:

- A network server that maintains mobile computing contexts within a client-specific domain;
- An asynchronous callback mechanism to notify Web clients when a user's dynamic computing environment changes;

- A syntax for referencing dynamic information in URLs and documents.

Representing mobile computing contexts. Mobisaic uses dynamic environments to represent a user's mobile computing context. The basic unit in a dynamic environment is the dynamic environment variable, which is conceptually similar to a standard shell environment variable: dynamic environment variables have a name and a value, and they can be accessed and changed to customize applications to the user's mobile computing environment just as shell environment variables customize applications launched from the shell. However, unlike shell environment variables, which are associated with a login process, dynamic environment variables are associated with users and places, and have indefinite lifetimes. Applications on the network with sufficient privilege can access and change dynamic environment variables, and whatever changes they make can be seen by other applications with sufficient access privileges.

Notification of changes in mobile computing contexts. Active documents allow environmental changes to be reflected in the information displayed to the user. If the information in an active document that the client is displaying becomes invalid, then the client can be notified of the change so that it can display a more relevant document. Client notifications contain the name of the variable that changed, and its new value.

For example, say that the user changes cell locations in the wireless environment. The wireless communications system that is monitoring the user's location can publish the new location by updating the Location variable in the user's dynamic environment. If the user were displaying a document that was sensitive to location, the client would have subscribed to the Location variable, and would receive a notification informing it of the change. At this point, the client could take action in response to the change, such as loading a new document that relates to the user's new location.

Syntax and scope. A Mobisaic client relies on a syntax for referencing dynamic environment variables within dynamic URLs and active documents. The syntax supported by Mobisaic is of the form $\$(e n-$ vironment.variable), where environment and variable denote a dynamic environment and dynamic environment variable, respectively. For example, \$(bershad.Location) would reference the name of Brian's current location in the wireless network. Mobisaic also supports a shorthand notation for referencing variables in the user's dynamic environment. If the reference doesn't contain the name of an environment, then Mobisaic assumes that the variable denoted is a reference to the environment associated with the user. Thus, \$(Location) refers to the Location environment variable in the user's dynamic environment.

Mobisaic supports recursive references to dynamic environment variables, so that environment or vari- 
able names can themselves be references to dynamic environment variables. For example, given that locations have dynamic environments associated with them, $\$$ (\$(bershad.Location).Printer) would reference the Printer variable in the environment associated with Brian's current location.

\section{$3 \quad$ Using dynamic URLs}

Dynamic URLs allow a single URL to return different documents or execute different commands depending upon the state of the user's dynamic environment at the time the query is executed. A URL is dynamic if it references at least one dynamic environment variable. For example, in our department we have written HTML documents describing ourselves and the places in which we work (see Figure 1). The name space of these documents on our server is well structured, enabling the following dynamic URL to return the document describing the user's current location:

\section{http://www/places/\$(Location).html ${ }^{1}$}

Another example of a dynamic URL is a Web server that has a program busroute which takes a starting location, a destination, and a time as arguments, and returns an HTML document detailing how to get to the closest bus stop on the shortest bus route to the destination. A dynamic URL to find the bus route to the Space Needle in Seattle would appear as:

http://www/htbin-post/voelker/busroute

?\$(Location)?SpaceNeedle?\$(Time.TIME)

Note that the question marks in the query are standard HTML syntax denoting the arguments that are passed to the program invoked on the server.

\subsection{Resolving dynamic URLs}

When a user selects a dynamic URL in a document, the client browser is responsible for resolving all references to dynamic environment variables within the URL. The client obtains the values of dynamic environment variables from the appropriate dynamic environment and replaces the references with the values as strings. When all variable references have been resolved, the result is a standard URL that the client then sends to the server. For example, if a user were in office 433 and selected the location description dynamic URL in the previous section, the client would resolve the Location dynamic environment variable in the user's context and send the resulting URL to the server:

\section{http://www/places/433.html}

Having the Mobisaic client resolve the dynamic environment variable references gives the most flexibility to the system. The variable references could have been resolved in two other places: the application, if the dynamic URL named a program on the server to execute,

\footnotetext{
${ }^{1}$ For brevity, we use www in place of our server at www.cs.washington.edu.
}

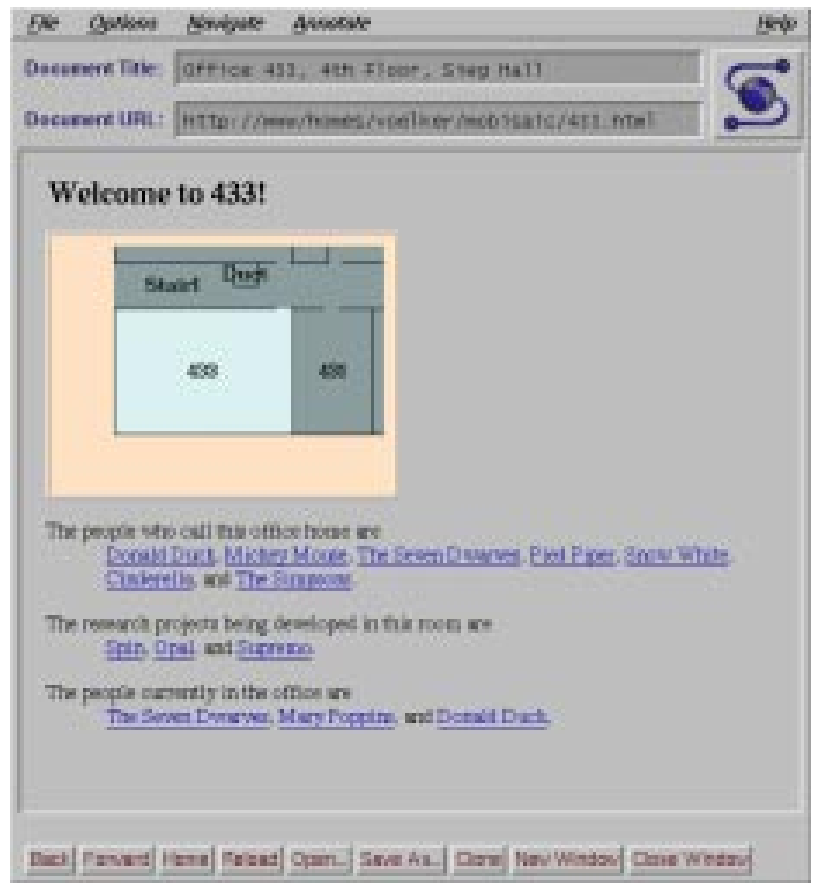

Figure 1: The WhereAmI active document, referenced by http://www/places/\$(Location).html

or the server. If applications had to resolve the variable references, then dynamic URLs would be limited to naming only those applications that were modified to understand and use dynamic environment variables. Likewise, if the server were to resolve the references, then dynamic URLs would be limited to using only dynamic environment aware servers. When the client resolves the references, however, dynamic URLs can evaluate to a URL that names any document or application on any server that a standard URL can name.

\section{Active documents}

Active documents are HyperText Markup Language documents that enable the Web client to automatically react to changes in a user's mobile computing context. They enable the client to be able to update the information being displayed without the user having to navigate the Web, and place less of a burden on the user to search for information by placing more of a burden on the author to organize it. This tradeoff is possible in a mobile environment because users who roam the wireless network are quite likely to be interested about who and what is in their immediate surroundings, and the information in a user's mobile context is enough to enable a Web client to do the searching on the user's behalf. In this way, it changes the way users interact with the Web: they spend less time searching for information because the client presents it to them as they interact with their surroundings.

This section describes how to write active documents and how the client handles them when they are 
being viewed by the user. To illustrate these processes, it also describes a set of pages collectively called the WhereAmI active document, which is a guide for visitors to our department. Each page in it corresponds to a place in our wireless network, and contains a brief description of the place, links to the home pages describing the occupants of the place, and links to pages describing any projects housed in the place. When a user selects this active document, the client loads the page corresponding to the user's location. Then, as the user changes rooms, the client automatically discards the page describing the old room and replaces it with the one describing the new room. Each page in the WhereAmI active document can be loaded using the dynamic URL from the previous section:

$$
\text { http://www/places/\$(Location).html }
$$

Authors write active documents just like they write standard HTML documents, with one addition. They must place a subscribe command in the document which lists the dynamic environment variables that the client must subscribe to when it loads the document. In effect, the variables listed in the subscribe command are the elements of a user's mobile context that, when they change value, invalidate the information in the document. The new values of the variables also provide the information necessary for the client to determine which document to load in place of the current one.

A subscribe command is embedded in an HTML comment line. By having the subscribe command embedded in a comment, active documents remain backwards compatible and can be loaded by standard Web clients that do not support the features of Mobisaic. The command has the following form:

$$
<!-(\text { subscribe to variable variable } \ldots)->
$$

Variable is a standard reference to a dynamic environment variable. When the client loads the document and parses subscribe commands, it subscribes to each variable specified in the command.

When the client receives a notification for a subscribed variable indicating that the variable has changed value, the new value of the variable in the notification determines what action the client will take in the face of this notification. The new value of the variable can be an explicit directive to the client:

reload Re-execute the URL that loaded the current document. If the URL is dynamic, the references to dynamic environment variables are resolved again.

load URL Execute a new URL and load the document in the same window.

spawn URL Execute a new URL and load the document in a new window.

close Close the current window.
Otherwise, the client does not interpret the new value and simply reloads the document. If the URL naming the document is a dynamic URL, then the client will evaluate the dynamic URL as if the user had selected it.

For the WhereAmI active document, each page has the following subscribe command:

$$
<\text { !- (subscribe to } \$(\text { Location }))->
$$

The commands tells the client that, when it loads the page, it should subscribe to the Location variable in the user's dynamic environment. When this variable changes value, the client will receive a notification with the new value of Location. Since the value is not an explicit command to the client, it will ignore the value itself and use the notification as a signal to reload the active document. And since the URL naming the document is dynamic, the client will re-evaluate it and load the active document that describes the user's new location.

\subsection{Arbitrary client notifications}

In addition to receiving notifications for any active documents the client is displaying, the client can also receive notifications that do not refer to any of its displayed documents. To receive such notifications, the client subscribes to the MOBISAIC-STREAM variable in the user's dynamic environment. Any process can then send notifications to the client to, for example, spawn a window to load a new document that might be of interest to the user.

\section{Mobisaic on the desktop}

Although Mobisaic was originally inspired for use in a mobile computing environment, it can also be useful in the desktop environment. There are a number of information sources in the WWW that produce information periodically, and it is straightforward to write documents in Mobisaic that tap into these sources. Some documents that have already been written include simple cron scripts that, early in the morning, publish the URLs for the Dr. Fun and Dilbert comic pages to a list of interested users running Mobisaic. The published URLs spawn new windows showing the contents of the pages. When users come in to work in the mornings, the daily comic pages are already showing on their screens.

As another example, we use active documents together with electronic mail to implement a distributed, recommendation-based "hot list" of new and interesting pages. Our local departmental version of Mosaic has been modified to make it easy to forward URLs to others in our department using email, so now it is common practice for people to forward onto friends URLs that they have discovered and find interesting. The email messages generated by Mosaic have a special mail tag, $X-U R L$, that contains the URL being forwarded. A user's incoming mail filter finds these tags and publish the URLs in them to the user's Web client. The result is that, when users have a URL forwarded to them, a window automatically appears 
on their screen displaying the document referenced by the URL.

A third application uses active documents to display and update stock quotes. A background filter monitors a stock quote information source, and, in a dynamic environment for stock prices, publishes the latest values of the stocks it monitors as they change. Documents displaying the information for a given stock subscribe to the dynamic environment variable associated with the stock in the stock dynamic environment. When users view a document describing or referencing a stock, the document will update itself as new stock values are published in the dynamic environment variable for stocks.

\section{Implementation}

This section discusses our implementation of Mobisaic and the changes we applied to a standard Web client to use them.

\subsection{Dynamic environments}

Dynamic environments [Schilit et al. 93B] support a network-based publish and subscribe paradigm [Oki et al. 93]. A dynamic environment is maintained by a subscription-based server to which applications broadcast queries and from which applications receive multicast notifications. We use the zephyr notification system [DellaFera et al. 88] to implement the publish and subscribe facilities. Zephyr allows programs to subscribe to any number of message classes and class instances to which other programs can address and send messages. Since multiple programs can subscribe to the same message class and class instance, a message to a class and instance will be multicast to all subscribing programs. A dynamic environment server is a zephyr client that listens to a specific zephyr message class for dynamic environments and a class instance that is the name of the environment it serves. Applications that interact with dynamic environments send zephyr messages with the dynamic environment message class, the name of the environment as the class instance, and the name of the dynamic environment variable as the recipient.

We chose to use zephyr because it provides network transparency, automatic subscriber-based routing, authentication, asynchronous notification, and the ability to run redundant zephyr clients supporting redundant instances of the same dynamic environment. (We presently do not take advantage of zephyr's authentication system or support for redundancy). Zephyr is not without its drawbacks, however. It operates only within relatively small administrative domains, such as a department or campus. It cannot distribute information quickly to many hosts, which can become a problem in the current system during heavy load. (This bottleneck and a possible solution to it are discussed in [Schilit and Theimer 94].) Fortunately, a C library interface hides the use of zephyr from Web clients, so changing to a new transport should be relatively easy.

\subsection{Client modifications}

Any Web browser can be modified to support dynamic URLs and active documents provided that it supports an interface for loading and reloading documents, spawning and closing windows, and the ability to add an asynchronous input descriptor to its set of inputs. A client library handles all communication with dynamic environments, and parses dynamic environment variable references. Filters, supplied by the library, are applied to the input and output communication paths. The filter on the output stream resolves references to dynamic environment variables embedded in dynamic queries, and the filter on the input stream subscribes the client to dynamic environment variables embedded in active documents. If the client supports an internal interface for document and window manipulation, then it can be directly linked with the Mobisaic client library. For those clients that only support an external interface or do not have an interface for adding asynchronous input descriptors, we have a wrapper program that handles dynamic environments and controls the Web client as a child process.

Our prototype Mobisaic client is the $\mathrm{X}$ Mosaic client [Andreessen and Bina 94] extended with the Mobisaic client library. The X Mosaic client has a relatively clean internal interface for loading documents and spawning windows, so most of the code changes were to add the file descriptor for the dynamic environment communication channel to the list of input descriptors, the callback to handle asynchronous input on the descriptor, and calls to the Mobisaic library filters on the input and output communication paths. Overall, our changes added less than 60 lines to the client.

Our infrastructure for wireless mobile computing is still under construction, but Mobisaic Web clients for two different environments are being developed. The first Mobisaic client will be the existing modified $\mathrm{X}$ Mosaic client running on a laptop running Linux. The laptop will use a Proxim RangeLan2 wireless ethernet PCMCIA card to communicate with the wireless network. An infrared receiver attached to the serial line detects transmissions from infrared beacons placed in rooms and hallways for fine-grained location information. The second Mobisaic Web client will be the $W^{*}$ Web client running in the Wit [Watson 94] environment.

\section{Summary}

This paper has described a World Wide Web information system called Mobisaic that investigates information browsing in a mobile wireless computing environment. Mobisaic introduces two mechanisms, dynamic URLs and active documents, for incorporating contextual information from a user's mobile computing environment into the Web. Dynamic URLs allow a single URL to return different documents or execute different commands depending upon the values of the embedded variables at the time the URL is selected by the user. Active documents use dynamic environment variables to have the client to subscribe to notifica- 
tions that invalidate their contents with respect to the current state of the user's mobile computing context.

Minimal modifications are required to Web clients and the URL syntax to support the features of Mobisaic. Web servers need no modifications whatsoever. A library hides the details of the communication mechanism and provides filters for parsing and resolving dynamic environment variable references, making it straightforward to modify a Web client to take advantage of the features of Mobisaic Web system.

\section{Acknowledgements}

We would like to thank Marc Fiuczynski, Ed Lazowska, Hank Levy, Stefan Savage, Terri Watson, George Forman, and John Zahorjan for their suggestions on Mobisaic. Bill Schilit at Xerox Parc has been incredibly helpful in discussing context-aware applications. Finally, special thanks are due to Xerox PARC for supplying us with the software and hardware that began our experiment in mobile computing.

\section{References}

[Andreessen and Bina 94] Marc Andreessen and Eric Bina. "NCSA Mosaic: A Global Hypermedia System" In Internet Research, 4(1):7-17, Spring 1994.

[Berners-Lee et al. 92] Tim Berners-Lee, Robert Cailliau, Jean-Francois Groff, and Bernard Pollermann. "World-Wide Web: The Information Universe" In Electronic Networking: Research, Applications, and Policy, 2(1): 52-58, Spring 1992.
[DellaFera et al. 88] C. Anthony DellaFera, Mark W. Eichen, Robert S. French, David C. Jedinsky, John T. Kohl, and William E. Sommerfeld. "The Zephyr Notification Service." In Proceedings of the USENIX 1988 Winter Conference, Winter 1988.

[Oki et al. 93] Brian Oki, Manfred Pfluegl, Alex Siegel, and Dale Skeen. "The Information Bus - An Architecture For Extensible Distributed Systems" In Proceedings of the Fourteenth ACM Symposium on Operating System Principles, December 1993.

[Schilit and Theimer 94] Bill N. Schilit and Marvin M. Theimer. "Disseminating Active Map Information to Mobile Hosts" To appear in IEEE Network, September, 1994.

[Schilit et al. 93A] Bill N. Schilit, Norman Adams, Rich Gold, Michael Tso, and Roy Want. "The ParcTab mobile computing system." In Proceedings of the Fourth Workshop on Workstation Operating Systems, pp. 34-39, October 1993.

[Schilit et al. 93B] Bill N. Schilit, Marvin Theimer, and Brent B. Welch. "Customizing mobile applications." In Proceedings of the USENIX Symposium on Mobile $\&$ Location-Independent Computing, pp. 129-139, August 1993.

[Watson 94] Terri Watson. "Application Design for Wireless Computing." In Proceedings of the Workshop on Mobile Computing Systems and Applications, November, 1994. To appear. 